\title{
Proving that ICFs overestimate the nitrogen abundances of FLIERs
}

\author{
Denise R. Gonçalves ${ }^{1}$, Barbara Ercolano ${ }^{2}$, A. Carnero ${ }^{2}$, \\ A. Mampaso ${ }^{3}$, R. L. M. Corradi ${ }^{3,4}$ \& M. J. Barlow ${ }^{2}$ \\ ${ }^{1}$ IAG - Universidade de São Paulo, Brazil; Email: denise@astro.iag.usp.br \\ ${ }^{2}$ Department of Physics and Astronomy, University College London, UK \\ ${ }^{3}$ Instituto de Astrofísica de Canarias, Spain; ${ }^{4}$ Isaac Newton Group of Telescopes, Spain
}

\begin{abstract}
We have recently (Gonçalves et al. 2006) shown that previously reported enhanced N abundances in fast, low-ionization emission regions (FLIERs) of PNe, particularly in NGC 7009, may be due to ionization effects. We reached this conclusion through the 3D MOCASSIN photoionization modeling of the bright inner rim of NGC 7009 and its pair of FLIERs, assuming homogeneous elemental abundances throughout the nebula, for $\mathrm{N}$ as well as other elements. The $\left(\mathrm{N}^{+} / \mathrm{N}\right) /\left(\mathrm{O}^{+} / \mathrm{O}\right)$ ratio predicted by our models is 0.60 for the rim and 0.72 for the knots, clearly in disagreement with the $\mathrm{N}^{+} / \mathrm{N}=\mathrm{O}^{+} / \mathrm{O}$ assumption of the ionization correction factors (ICF) method. Therefore the ICFs are underestimated in both components, rim and knots, but more so in the knots. This effect is partly responsible for the apparent $\mathrm{N}$ overabundance claimed for FLIERs.

Based on the above results we are also investigating what would be the effect of the N/O ICF overestimation on the definition of Type I PNe which do not have FLIERs-like structures.
\end{abstract}

Keywords. ISM: abundances - planetary nebulae: individual (NGC 7009) - jets and outflows

\section{The ICF apparent N-enhanced FLIERs}

Fig.1 shows the different structures of NGC 7009 in the observed (left) and modeled (right) [N II] images. In Fig.2 we have an example of the apparent N-enrichment in FLIERs obtained with the ICF method (Balick et al. 1994; Gonçalves et al. 2003) - not reliable for spatially resolved PNe (Alexander \& Balick 1997; Gruenwald \& Viegas 1998). This apparent $\mathrm{N}$ enhancement -up to a factor of 2, from the plots in Fig.2- has been interpreted as an evidence that FLIERs originate from recent high-velocity ejections of the PN central star. The application of ICF-based methods may not be suitable for the interpretation of long-slit spatially resolved data, hence hiding the true nature of FLIERs.
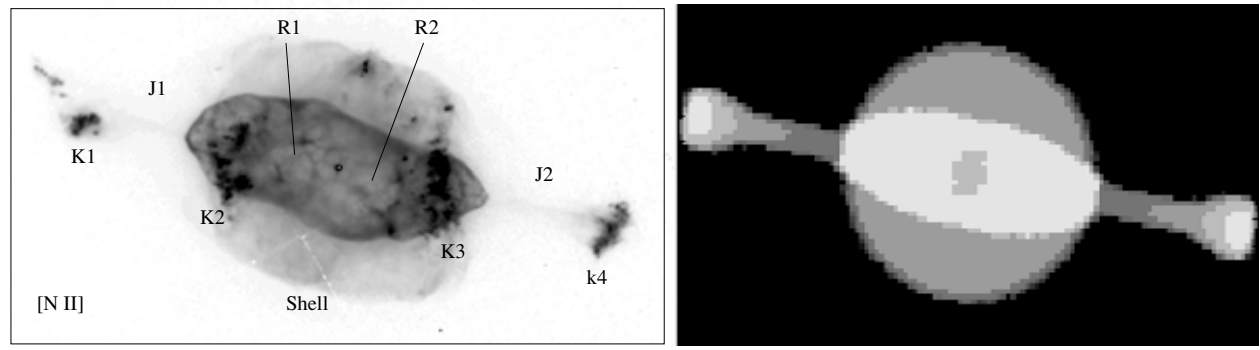

Figure 1. Left: HST [N II] image of NGC 7009, in logarithmic scale, in which labels mark the positions of the outer $(\mathrm{K} 1, \mathrm{~K} 4)$ and inner $(\mathrm{K} 2, \mathrm{~K} 3)$ pair of knots, the pair of jets (J1, J2), the $\operatorname{rim}(\mathrm{R} 1, \mathrm{R} 2)$ and the shell. The size of the box is $65 \times 32$ arcsec. Right: projected [N II] emission map from the model, in arbitrary units. 


\section{MOCASSIN modelling of NGC 7009}

The nebula was modeled using the 3D photoinisation code, MOCAssin, of Ercolano et al. (2003), which is geometry-independent, and can self-consistently treat the transfer of the diffuse component. We constructed a model, aiming at reproducing the observed geometry and spectroscopic "peculiarities" of a PN resembling NGC 7009, to explore the possibility that the enhanced $[\mathrm{N}$ II] emission observed in the outer knots may be due to ionization effects. The model is constrained by the HST [O III] and [N II] images as well as Isaac Newton Telescope long-slit, intermediate dispersion spectra, along the PN major axis. See Gonçalves et al. (2003).

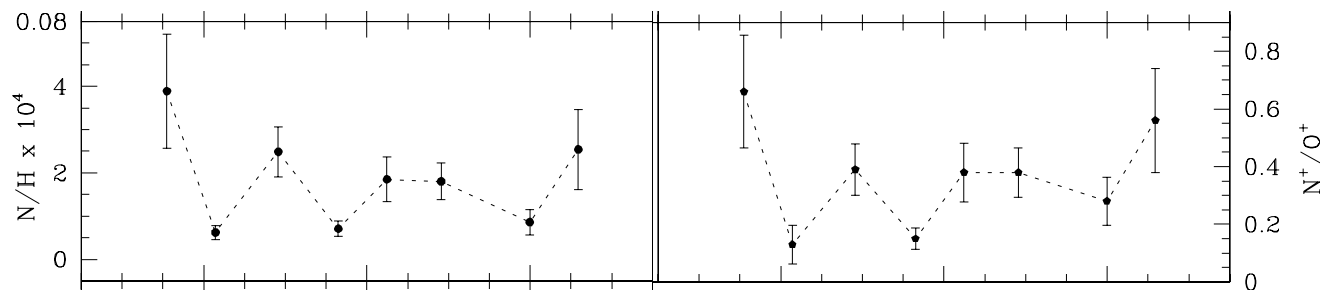

Figure 2. Abundance profiles for NGC 7009. K1 is to the left and K4 to the right, as in Fig.1 Left: The ICF total nitrogen abundance. Right: The once-ionized nitrogen over oxygen ratio.

The simplest possible density distribution of our model included an elliptical rim, a sphericall shell, cylindrical jets and disk-shaped outer knots (FLIERs), as shown in Fig.1. The distance adopted is $0.86 \pm 0.34 \mathrm{kpc}$ (Fernández et al. 2004). The stellar temperature and luminosity of NGC 7009 are relatively well known: $\mathrm{T}_{\text {eff }}=80,000 \mathrm{~K}$ and $\log \mathrm{L}_{*}=3.50$. Using the $\mathrm{D}$ above and the observed size of the nebula, the outermost knots of the model extend up to $3.88 \times 10^{17} \mathrm{~cm}$. The best model that fits the spectra of the different nebular structures, as well as the $[\mathrm{O} \mathrm{III}]$ and $[\mathrm{N} \mathrm{II}]$ images, has homogeneous abundances of 0.11 , $3.2 \times 10^{-4}, 2.0 \times 10^{-4}, 4.5 \times 10^{-4}, 1.06 \times 10^{-4}, 9.0 \times 10^{-6}$ and $1.2 \times 10^{-6}$, for He, C, $\mathrm{N}, \mathrm{O}, \mathrm{Ne}, \mathrm{S}$ and Ar, respectively. These abundances are very similar to those derived empirically for the entire nebula in Gonçalves et al. (2003).

\section{Acknowledgements}

DRG and BE would like to thank support of the Brazilian Agency FAPESP (03/096920, 04/11837-0). We also acknowledge the partial support of the Spanish Ministry of Science and Technology (AYA 2002-0883) and the organizers for the IAU grants.

\section{References}

Alexander J., \& Balick B. 1997, AJ 114, 713

Balick B., et al. 1994, ApJ 424, 800

Ercolano B., Barlow M. J., Storey P. J., \& Liu X.-W. 2003, MNRAS 340, 1136

Fernández R., Monteiro H., \& Schwarz H. E. 2004, ApJ 603, 595

Gonçalves D. R., Corradi R. L. M., Mampaso A., \& Perinotto M. 2003, ApJ 597, 975

Gonçalves D. R., Ercolano B., Carnero A., Mampaso A., \& Corradi R. L. M. 2006, MNRAS 365,1039

Gruenwald R., \& Viegas S. M. 1998, ApJ 501, 221 